\title{
Beziehungen zwischen Arzt und Apotheker im 16. bis 18. Jahrhundert in der Schweiz
}

Von Hans-Rudolf Fehlmann

\section{Einleitung}

Die Ärzteschule von Salerno in Süditalien, deren Gründungszeit unbekannt ist - bereits 846 wurde sie als «alt» bezeichnet -, vermittelte durch die Übersetzungen aus dem Arabischen eines Constantinus Africanus (11. Jahrhundert) den Studenten das medizinische Wissensgut von Hippokrates über Galen bis zu den sogenannten Arabischen Ärzten. Unter diesen berühmten salernitanischen Arzneibüchern nahm das Lehrgedicht Regimen sanitatis salernitanum eine besondere Stelle ein.

Bis zum Hochmittelalter gab es weder eine Scheidung des ärztlichen und pharmazeutischen Berufes noch genaue Ausbildungsvorschriften. Im Jahre 1224 wurde die Universität von Neapel und 1289 diejenige von Montpellier, im 15. und 16. Jahrhundert die bekannteste europäische Medizinhochschule, gegründet. Wohl unter dem Einfluß dieser ersten medizinischen Zentren kristallisierten sich schon anfangs des 13. Jahrhunderts die beiden Medizinalberufe langsam als solche heraus, so daß es einer geschichtlichen Folgerung entsprach, daß Kaiser Friedrich II. von Hohenstaufen (1194-1250) für sein Königreich Sizilien die erste umfassende Medizinalverordnung im Jahre 1240 erließ. Deren wichtigste Verfügungen waren:

- Beaufsichtigung der Apotheken und der Arzneibereitung in denselben durch vorschriftsmäßig ausgebildete und staatlich approbierte Ärzte;

- Verbot des Apothekenbesitzes für Ärzte;

- Verbot geschäftlicher Gemeinschaft zwischen Ärzten und Apothekern;

- Vereidigung der Apotheker, Verpflichtung nach speziellen ärztlichen oder anerkannten allgemeinen Vorschriften zu arbeiten;

- amtlich festgesetzte Arzneimittelpreise;

- Beschränkung der Errichtung von Apotheken auf bestimmte Orte nach staatlicher Genehmigung.

Die Verordnung Friedrich II. wurde für die meisten späteren Medizinalgesetze richtungweisend. Die Entwicklung dieser Scheidung von Arzt- und Apothekerberuf setzte sich, vor allem im Norden Europas, nicht ohne Widerstand und nur langsam durch. In der Schweiz, die mit ihren kanto- 
nalen Sanitätsgesetzen eine nationale Lösung nicht zuläßt, ist diese Entwicklung noch am Ende des 20. Jahrhunderts nicht abgeschlossen; es scheint sogar, daß man weiter davon entfernt ist als vor einigen Jahrhunderten, auf eine vernünftige gesetzliche Grundlage zu kommen.

\section{Das Arzt-Apotheker-Verhältnis im 16. und 17. Jahrhundert}

Immer und überall in der Schweiz kam es vor, daß Ärzte sich im Apothekerberuf betätigten und umgekehrt. Die Grenze gewollter und staatlich tolerierter, ebenso ungewollter Übergriffe wird nie scharf gezogen werden können, da hier zu viele Faktoren eine Rolle spielen. Dort, wo sich zwei Berufe, die im Grunde genommen das gleiche Ziel verfolgen, in ihren Kompetenzen überlappen - und dies ist der Fall bei der ärztlichen Medikamentenabgabe -- gab es und wird es immer Friktionen geben.

Es sind schriftliche Dokumente vorhanden, die nicht nur Ausnahmefälle gestatteten, sondern zum Beispiel den Apothekern das Recht des Kurierens ausdrücklich einräumten für den Fall, daß kein Arzt zur Hand war. So schreibt die Luzernische Verordnung von 1592 für ihren Stadtarzt vor: «Er soll mit dem Apotheker die Kranken besuchen, in schweren und langsamen Kuren dem Apotheker auch Underricht und Instruction geben, wie man procediren solle... sonderlich wo der Doctor über Feld reysen oder lybshalb nit woll uff wäre oder nüt wolle.»

Ein Gegenbeispiel, wonach ein Arzt mit einer eigenen öffentlichen Apotheke in die angestammte Sphäre des Pharmazeuten eingreift, liefert die Stadt Biel im Jahre 1677: «Der Arzt Dr.Grosjean hat eine öffentliche Apotheke errichtet. Der Rat findet, da Grosjean medicinam studiert und zum Stadtmedicus aufgenommen sei, und überdies zwei Apotheken in hiesiger Stadt nicht bestehen mögen, so soll er die seinige aufgeben, sich mit seiner Stelle begnügen und den bestellten Apotheker entlassen innerhalb sechs Monat.» Ein Jahr später bezeugt ein bei der Medizinischen Fakultät der Universität Basel eingeholtes Gutachten, «daß Dr. Grosjean nicht befugt sei, eine Apotheke zu halten, jedoch dürfe er die Medikamente für seine Kranken selbst oder durch einen Diener machen lassen. Seckelmeister Roter protestiert dagegen, 〈das sye gegen die fürgelegte Reichsordnung〉.»

In Pestzeiten, so z.B. als im Jahre 1519 diese Krankheit die Stadt St.Gallen heimsuchte, waren die Ärzte sogar froh, auf die Mitarbeit der Apotheker zählen zu können. Joachim Vadian (1484-1551), Reformator und seit 1518 Stadtarzt in St.Gallen, floh im darauffolgenden Jahre nach 
Zürich, nachdem er den St. Galler Apotheker Matthias Oswald (1482-1587) mit der Lieferung der Medikamente an die Pestkranken beauftragt hatte. Bernhard Milt schreibt dazu weiter, daß das Verhältnis zwischen Arzt und Apotheker ein gutes gewesen sein müsse, denn «1526 wandte sich Oswald schriftlich an den Stadtarzt, weil nach dem Tode von dessen Bruder die Erbgemeinschaft seine Rechnung für gelieferte Medikamente nicht bezahlt hatte. Oswald bemerkte dabei aber, daß er lieber auf dieses Geld verzichte, falls eine Intervention Vadian Unannehmlichkeiten bereiten könnte: «dan ewer wirde dient mer teglichen in mannicher sachen und handeln»». ${ }^{1}$

Nach Vadians Tod anerbot sich der Apotheker, wieder ärztlichen Dienst in der Stadt zu versehen. «Es wurde aber am 15. April 1551 beschlossen, daß mine Herren jn nit ghaißen haben wellen und jm ouch nützid verbietend, er artzne, sech den bronnen oder nit». ${ }^{1}$

Der große Arzt und Naturphilosoph Theophrastus Bombastus von Hohenheim, genannt Paracelsus (1493-1541), brachte nicht nur den damaligen Apothekern, sondern vor allem seinen Kollegen von der medizinischen Fakultät Mißtrauen entgegen. In der Paracelsus zugeschriebenen Eingabe an den Basler Rat (1527), in welcher er gegen die Wirrnisse der bestehenden Medizinalverordnungen aufgetreten ist, visiert der Hohenheimer folgende Zustände ${ }^{2}$ an: «Er weist auf die Mißstände im städtischen Apothekerwesen und deren Beseitigung durch Prüfung der Apotheker, Kontrolle der Rezepte, regelmäßige Visitationen der Apotheken, Aufstellung einer Taxe und Verbot gegenseitiger Gewinnbeteiligung zwischen Ärzten und Apothekern hin.»

Die von Paracelsus in der «De gradibus»-Vorlesung an der Universität Basel an seinen Berufskollegen geübte Kritik hat folgenden Wortlaut: «Wenn ich Euch Doktoren nicht gefallen sollte, so denkt daran, daß Ihr mir auch nicht gefällt, und daß Ihr in Euren Werken und Handlungen Blinde sind, ohne alle Würdigkeit Eures Standes. Ihr habt den Namen, aber nicht das Werk. Keiner von Euch sollte Arzt genannt werden, sondern Kopisten und Nachahmer des Avicenna und Galen, die sonst nichts vermögen.» ${ }^{3}$

Die zeitgenössische Rezeptierart der Basler Ärzte wird um 1550 vom Basler Apotheker Balthasar Hummel, einem Studienkollegen von Felix Platter in Montpellier, scharf kritisiert: «Ich beklage mich, denn ich habe kein vertrib in der Apoteck; man ordiniert gar wenig, man halte nüt ze Basel uf geschickte artzet, man schreib mer Teutsche recept, dan Latine. Die medici richten der mertheil purgierung mit dem senet (= Folium sennae) aus, siensholtz (= Süßholz) und ander narrenwerch. D. Isaac (Dr. Isaac Keller, 
Professor der Medizin, Basel) mach selber den krancken gmein lumpenwerch (= Selbstdispensation). Ich wollt liber ein bettelvogt zu Basel sein, dan ein Apotecker.» ${ }^{4}$ Auf dieses geharnischte Schreiben Hummels antwortet Felix Platter aus Montpellier: «Sy kennen nüt, die medici, dan purgieren, brauchen keine rechtschaffne remedia, wie ze Mompelier.» ${ }^{5}$

Aus einem weiteren Brief Hummels geht hervor, daß Felix Platter ihm in Aussicht stellt, er werde nach Beendigung seiner Studien an der Universität von Montpellier nach Basel kommen und neue, bessere Arzneivorschriften mitbringen. Sieht man sich diese, wie er sie z. B. in Praxeos ${ }^{6}$ niedergeschrieben hat, etwas genauer an, gibt es viele Vorschriften darunter, die man schon im Grabbadin von Mesue ${ }^{7}$ findet.

\section{Wie waren die Beziehungen im 18. Jahrhundert?}

Im Jahre 1733 will Luzern, neben Schaffhausen, Zürich und Bern, von Basel wissen, welche Ordnung «in Zubereitung und Verkaufung der Medicamenten zwüschen den allhiesigen Herren Medicis und Apoteceren observieret» werde $^{8}$. Aus Gerechtigkeitssinn heraus befragte der Stadtrat zunächst die Ärzte und Apotheker, erst dann ließ er vom Dekan der Medizinischen Fakultät der Universität Basel, Professor Johann Rudolf Zwinger (1692-1777), Hochschullehrer für praktische Medizin, eine eingehend gehaltene Antwort ausarbeiten.

Die Stellungnahme sämtlicher Apotheker Basels bezog sich auf die Gewissenhaftigkeit, die sie der Anschaffung, Lagerung, Kontrolle und Abgabe «aller erforderlichen Simplicia, Composita tam Chymica quam Galenica Medicamenta» entgegenbrachten. Durch eine Selbstdispensation der hiesigen Ärzte würden den Pharmazeuten «großen Schaden und Ruin erwachsen». Sodann wären «die Herren Medici mit ihren Haus Apothecken und den darinnen befindlichen Medicamenten nicht im stand, den Patienten mit den nötigen Hilfs Mitteln beyzuspringen». Der Brief an den Rat schließt mit der Feststellung, daß «fast aller orthen die Herren Medici Thre Praxin, hingegen die Apothecker Ihr Profession exerciren, und keinem erlaubt, darinnen eingriff zu thun».

Die Antwort des Herrn Dekans Zwinger ergeht sich zuerst in würdigen und würdigenden Kontemplationen über den Gang der «weitläufigen Medizinischen Wissenschaft» und ist gespickt mit Belegen der großen antiken Ärzte über die Ernährungsgewohnheiten, die mit der Zeit zu «Trägheit und Müssiggang» und dadurch zu allerhand «Überfluß in Essen 
und Trincken» geführt hätten. Wie aktuell lassen sich diese Worte anhören! «Nach und nach seien die Menschlichen Leiber schwächer und damit die Anfälligkeit für Krankheiten größer geworden. Das habe zu einer übermäßigen Vermehrung der Medikamente geführt, wodurch denen Medicis so viel arbeit auf den Hals gewachsen sei, daß Sie nicht mehr alles selbst bereithen können. Daher hätten sie das Geschäft, die arzneyen zu bereiten, anderen hiezu Tauglichen gern überlassen, und dies sei der Beginn der ApotheckerKunst gewesen (sic!)». Sodann zitiert Professor Zwinger vor allem deutsche Vorschriften, die auf Trennung der beiden Medizinalberufe pochen, und er bringt einen Exkurs aus einer zeitgenössischen medizinalpolitischen Schrift, worin eindeutig die Gründe und Konsequenzen der getrennten Berufsausübung bezeugt sind. Ohne aber eine klare Stellung zu beziehen, empfiehlt Zwinger schließlich den beiden Parteien, wie bis anhin «ohne Streit» fortzufahren und einander eher zu unterstützen. Wörtlich meint der Herr Dekan: «Gleichwie aber dem gemeinen wesen am besten gedienet, wann jegliche Profession in seine gebührende Schranken gesetzt, und ob denen einmahlen heilsam eingeführten Ordnungen gehalten wird, also entstehet im Gegentheil dem publico nicht geringes Unheil, wann kein unterschied der Professionen observiret, und ein jeder was ihne nur gelustet, oder worzu ihne sein privat Nutzen verlaitet, ohne Scheü unterfanget.»

Es fällt auf, daß Zwinger mit keinem Wort die Basler Medizinalverordnungen erwähnt, die alle die Trennung des Arzt- und Apothekerberufes gefordert haben.

Zum Schluß sei «kuriositätshalber» auf eine Situation Arzt - Apotheker hingewiesen, die wir gegen Ende des 18. Jahrhunderts in einer, damals bernischen Kleinstadt, in Aarau, antreffen: In der Mitte dieses Jahrhunderts bestanden mindestens zwei Apotheken in Aaraus Altstadt, diejenige des Samuel Imhof (1681-1751) an der Kronengasse gelegen ${ }^{9}$, und jene des Wilhelm Wydler (geb. 1730), damals Marktgasse, heute Rathausgasse. Zu jener Zeit gab es auch in Aarau Apotheker-Ärzte und Arzt-Apotheker, wie z.B. den Ratsherren, Arzt und Apotheker, Franz Daniel Rudolf Vögeli (1725-1783); Daniel Wassmer, Dr. med., Apotheker, des Regiments ${ }^{10}$, sowie weitere Ärzte und Wundärzte (Chirurgi). Die meisten waren politisch tätig, saßen im Rat oder später in den helvetischen Munizipalbehörden. Infolge mangelnder Quellenlage ist nicht auszumachen, ob und wo in Aarau diese Medizinalpersonen eine Apotheke oder eine eigene Praxis führten. Auf jeden Fall dispensierten die Ärzte und vor allem die Wundärzte Medikamente.

Der erwähnte Wilhelm Wydler, Posthalter und Apotheker zu Aarau, 
praktizierte seit 1758 neben seiner ungefähr um 1752 eröffneten Apotheke als «Medicus», ohne dafür ein Patent der Prüfungsbehörde besessen zu haben. Als Stadt im Kanton Bern unterstand Aarau der Bernischen Sanitätsgesetzgebung ${ }^{11}$. «Das Collegium Insulanum, die Gesamtheit der im Bernischen Inselspital tätigen Medizinalpersonen, hatte seit 1733 die Befugnis, die damals neu aufkommende Kategorie der Landärzte ${ }^{12}$ zu examinieren, wonach sie dann zur Ausübung ihrer Praxis in kleineren Orten und auf dem Lande befugt waren. Für die Wundärzte setzte der Sanitätsrat erst im Jahre 1788 durch, daß die Prüfung nicht mehr wie bis anhin durch eine 〈Meisterschaft,, sondern durch das Inselkollegium zu erfolgen hatte, worauf die Patente durch den Sanitätsrat erteilt wurden.»

Vor diesem Collegium Insulanum erschien nun am 23. Dezember 1782 der erwähnte Apotheker Wilhelm Wydler, um auf eigenes Begehren «über die einem Medico nöthigen Kenntnisse in der Arzneikunst, sowohl in der Anatomey als über die Art, innerlicheKrankheiten zu kurieren, behörigermaßen befragt» zu werden ${ }^{13}$. «Selbigen aber in beyden so schwach befunden, daß Wohldieselben (= Inselkollegium) nicht anders können, als Euer Wohledelgebohren (= Sanitätsrat) anzurathen, ihme die Bewilligung, als Medicus zu practicieren abzuschlagen, und ihm anzuhalten, sich mit seiner Profession als Apotheker zu begnügen. Indessen können meine gnädigen Herren nicht anders als sehr verwundert seyn, daß ihme der Magistrat zu Aarau nur erst nach deme er 24 Jahr lang als Medicus practiciert, die Praxis in der Arzneykunst verbietet. Und da er sich beklagı, daß weilen die Herren Medici und Chirurgi zu Aarau nun eigene Apotheken in ihren Wohnungen errichtet, er als bloßer Apotheker keinen oder einen gar kleinen Verdienst mehr hätte. So glauben meine gnädigen Herren der Billichkeit gemäß, daß womöglich denen Herren Medicis und Chirurgis Errichtung dergleichen eigenen Apotheken verboten werde. Actum den 23. December 1782.»

Eine Notiz im «Verzeichnis aller Ärzte, Wundärzte etc. der deutschen Lande des Standes Bern» aus dem Jahre $1787^{1+}$ führt in der Kategorie «Apotheker» auf: «Wilhelm Wydler, Stadt Aarau, Von wem patentiert?: nach bisheriger Gewohnheit ${ }^{15}$ wurde er von «Niemandem» patentiert, er besitzt lediglich einen 〈Lehrbrief〉.»

Diese Episode wirft ein grelles Licht auf die damaligen ungenügenden sanitätspolizeilichen Vorschriften in der Patent-Erteilung an Vedizinalpersonen. «Die vielen Ärzte, welche das kleine Städtchen Aarau damals zählte, wußten, vereint mit dem Únabhängigkeitsstreben der Stadtväter, jede einschränkende Maßnahme zu verhindern», ${ }^{16}$ und dies solange. bis endlich 
kurz nach der Gründung des Kantons Aargau im Jahre 1803, am 9. Januar 1804, die «Organisation des Sanitätswesens» klare, eindeutige Verhältnisse und Abgrenzungen zwischen den beiden Berufen der Ärzte und Apotheker herbeiführte ${ }^{17}$.

\section{Schlußbemerkungen}

Obwohl die Constitutiones von Kaiser Friedrich II. aus dem Jahre 1240 in den meisten europäischen Staaten für die Medizinalgesetze als Grundlage dienten, wurden sie von den Sanitätsbehörden eher nach freiem Ermessen und ohne rigorose Kontrolle gehandhabt.

Anhand einiger ausgewählter Beispiele aus dem 16. bis 18. Jahrhundert wird versucht, zu zeigen, daß Zeitströmungen, Krankheiten oder Epidemien wie auch politische Faktoren einen größeren Einfluß auf die Beziehungen zwischen Ärzten und Apothekern ausgeübt haben als eine konsequente obrigkeitliche Durchsetzung der Vorschriften.

Die betreffende Situation des Kantons Bern im 18. Jahrhundert ist dafür besonders charakteristisch. Noch in den dreißiger Jahren bestand, sowohl für die «welschen» als auch für die «deutschen Lande» des Bernischen Hohheitsgebietes, keine Verordnung für die Examinierung, Visitation und offizielle Verkaufstaxe für Apotheker, und keine «hochobrigkeitliche Prüfung» für Ärzte und W undärzte. Für die letzteren und für die Pharmazeuten wurden erst im Jahre 1788 strenge Vorschriften erlassen.

Allerdings konnten und können auch heute diese verbesserten Medizinalgesetze nicht verhindern, daß bei den Berufen der Ärzte und Apotheker Überschneidungen vorkommen, die sich trotz abgegrenzten Ausbildungsgrundsätzen in praxi immer wieder ergeben. Es wäre wohl vernünftiger, zu versuchen, diesen Kompetenzenstreit an der «Basis» durch längst postulierte gemeinsame Vorlesungen in Deontologie zu Beginn der beiden Studienrichtungen aus der Welt zu schaffen, als sich einem staatlichen Eingriff beugen zu müssen.

\section{Anmerkungen}

${ }^{1}$ Bernhard Milt, Vadian als Arzt, Verlag der Fehr'schen Buchhandlung, St.Gallen 1959, 101-103; Vera Waldis, Pest und Protestantismus, NZZ, Nr. 105, 8./9. Mai 1982.

${ }^{2}$ Robert-Henri Blaser, Paracelsus in Basel, Festschrift für Prof.Dr.phil. R. H.Blaser zum 60.Geburtstag, hrsg. von der Schweizerischen Paracelsus-Gesellschaft, St. Arbogast-Verlag, Muttenz/Basel 1979, 50/51 (Anm.68/69) und 121/118. 
${ }^{3}$ Paracelsus, Sämtliche Werke, hrsg. von Karl Sudhoff, München 1922-1933, I/4, 80.

1 J.A. Häfliger, Das Apothekenwesen Basels, Basel 1937, S.38, 63/64, 267/268.

5 Handgeschriebene Briefe Balthasar Hummels an Felix Platter um 1555, in der Universitätsbibliothek Basel, Signatur: Fr. Gr. I.8, fol. 148-161 (nicht transkribiert, unediert).

${ }^{6}$ Felicis Plateri Archiatri et Profes. Basil ... Praxeos ... Tractatus secundus, Libro uno, Basileae, Typis Conradi Waldkirchii, M. DCIII: z. B. Unguentum aegyptiacum (S.966) ist im Grabbadin (Anm. 7) auf S.252 aufgeführt!

' L.J.Vandewiele, De Grabadin van Pseudo-Mesues (XI"-XIJ" eeuw (= Jahrhundert), Diss. Gent 1962, 252 et al.

s J.A. Häfliger, a.a.O., S. 177-181.

" im Katasterplan von Aarau 1824. (Stadtarchiv Aarau) ist sie als Apotheke cingezeichnet; schriftliche Quellen konnten bis jetzt nicht gefunden werden.

${ }^{10}$ Erich Bayer, Wörterbuch zur Geschichte, A. Kröner Verlag, Stuttgart 1960, S.411, Sp.2: Regiment: ... Obrigkeit; Hof-, Reichs- oder Stadtrat ...

11 a) Verordnung (VO) für sämtliche Apotheker zu Stadt und Land vom 22.2.1788, Instructionen Manual des San. Rates, Staatsarchiv Bern, B XI, 9, 99-110.

b) Eine frühere VO von 1738 ließ die Apotheken auf dem «Land» nicht visitieren, sie brauchten keine Betricbsbewilligung, es fand keine Examinierung der Apotheker statt, und keine Taxe wurde verlangt.

12600 Jahre Inselspital, 1354-1954, verfaßt von Hermann Rennefahrt und Erich Hintzsche, Hans Huber, Bern 1954, 298.

13 Instructionen Manual des San. Rates, a.a.O., St A Bern, B III, 130.

14 Staatsarchiv Bern. B XI, 320.

1.5 $\mathrm{Vgl}$. Anm. $11 \mathrm{~b}$.

16 Aarauer Altstadtapotheken, Martin Pestalozzi und Heinrich Göldlin, Eigenverlag H. Göldlin, Apotheke, Rathausgasse 29, Aarau 1979, S.5.

17 Hans-Rudolf Fehlmann, Die erste Aargauer Apothekertaxe. Schweiz. Apothekerzeitung (SAZ) 111 (1973) 436-438: und Conrad Meier, Das aargauische Sanitätsgesetz, SAZ 103 (1965) 355

\section{Summary}

This contribution wants to show by choosed examples from the 161 h to the 181 h century that some epoch-trends (Zeitströmungen!), sicknesses or epidemic diseases, and also political facts did exercise a greater influence on the relations between the doctors and the pharmacists, than a consequent obedience of the corresponding laws.

We write down a characteristic situation from the $18^{\text {th }}$ century in the canton of Berne. Then neither the physicians nor the pharmacists had to undergo rigorous examinations by a sanitary magistracy. Accordingly there were often both profescions side by side in the same consulting room, or a practising doctor exercised also the pharmaceutical profession and viceversa. Only the sanitary law of 1788 brought severe examinations and a better delimination but not a perfect in Switzerland!- between the two medical professions.

Dr.phil. Hans-Rudolf Fehlmann, SchloBapotheke, CH-5103 Wildegg 